\title{
Cerebral Amyloid Angiopathy Presenting with Synchronous Bilateral Intracerebral Macrohemorrhages
}

\author{
Jeremy S. Shelton ${ }^{a} \quad$ Larry T. Davis $^{b} \quad$ Ray Stokes Peebles ${ }^{c}$ \\ Benjamin F. Tillman ${ }^{c}$ Bret C. Mobley ${ }^{a}$ \\ Departments of ${ }^{a}$ Pathology, Microbiology and Immunology, ${ }^{b}$ Radiology and Radiologic \\ Sciences and ${ }^{C}$ Medicine, Vanderbilt University Medical Center, Nashville, Tenn., USA
}

\section{Key Words}

Cerebral amyloid angiopathy · Synchronous bilateral intracerebral macrohemorrhages

\begin{abstract}
Cerebral amyloid angiopathy (CAA) is the deposition of amyloid proteins in the cerebrovasculature, which can lead to intracerebral hemorrhage. Intracerebral hemorrhage in CAA often presents with microhemorrhages and, less frequently, with more devastating macrohemorrhages. We present a case of CAA-related synchronous bilateral intracerebral macrohemorrhage which, to our knowledge, has yet to be reported in the literature, and postulate its relationship to antiplatelet therapy and transient elevations in blood pressure.
\end{abstract}

\section{Introduction}

Cerebral amyloid angiopathy (CAA) is the deposition of amyloid proteins in the cerebrovasculature. CAA is a well-known and relatively common etiology for intracerebral hemorrhage (ICH), with a predilection for the meningocortical vasculature $[1,2]$. In the elderly, CAA is the second most common cause of nontraumatic ICH after hypertension [3, 4]. While CAA is a relatively frequent cause of ICH, large synchronous bilateral lobar hemorrhage secondary to CAA is a rare but devastating event, which may be related to transient elevations in blood pressure and aspirin use. 


\section{Case Reports in Neurology}

Shelton et al.: Cerebral Amyloid Angiopathy Presenting with Synchronous Bilateral Intracerebral Macrohemorrhages

\section{Case Report}

A previously healthy 68-year-old woman awoke in the morning with dizziness which, by early evening, progressed to nausea, vomiting and a severe headache. Within $60 \mathrm{~min}$ of headache onset she became confused and somnolent. She had no history of hypertension, coagulopathy or illicit drug use. There was a history of low-dose aspirin use, which had reportedly been taken the day prior to the event. Upon initial presentation the patient's blood pressure was $178 / 70 \mathrm{~mm} \mathrm{Hg}$ and pulse $72 \mathrm{bpm}$. A CT scan displayed intraparenchymal hemorrhages involving the left parietal and bilateral temporal lobes and a 3-mm rightward midline shift. Approximately $3 \mathrm{~h}$ after headache onset, the patient's blood pressure was $189 / 68 \mathrm{~mm} \mathrm{Hg}$, pulse $59 \mathrm{bpm}$ and respiratory rate 9/min. MRI showed large areas of parenchymal hemorrhage in the right and left temporal lobes, the left parietal region and the right tentorium (fig. 1). Small foci of restricted diffusion near the vertex and the posterior limb of the internal capsule were present. There was no abnormal enhancement following contrast administration. No source of hemorrhage was identified on cerebral angiography. Transthoracic echocardiography did not reveal valvular pathology. Blood cultures were negative. A brain biopsy was planned but not performed due to brain herniation and a worsening neurologic examination. The patient died on hospital day four.

A complete autopsy was performed. Gross neuropathologic examination revealed intraparenchymal hemorrhage involving the left and right temporal lobes, softening of the medial temporal lobes secondary to transtentorial herniation and punctate hemorrhages in the superior portion of the left temporal lobe. Microscopic examination of the brain showed hemorrhagic infarctions within the bilateral temporal lobes, the right frontal and parietal lobes and the left occipital lobe. Thickened, hyalinized blood vessels were noted in the frontal, temporal, parietal and occipital lobes. Congo red stain and beta-amyloid immunohistochemical stains displayed multiple congophilic and beta-amyloid-reactive blood vessels (fig. 2) as well as neuritic plaques. Blood vessels adjacent to regions of hemorrhage showed microangiopathic change, with fibrinoid necrosis in addition to hyalinosis of the vessel walls. While no definite parenchymal microhemorrhages were identified by imaging, occasional foci of perivascular hemosiderin deposition with spongiosis indicative of prior hemorrhage were found on microscopic examination. Vascular malformations, onion-skin-type thickening or lipohyalinosis characteristic of hypertensive microangiopathy and saccular aneurysms were not identified. There were not significant numbers of perivascular inflammatory cells, providing no support for vasculitis. Grocott methenamine silver stain and Gram stain were negative for microorganisms. PCR of brain tissue did not detect the presence of HSV-1 or HSV-2 nucleic acid. Culture of brain tissue and immunohistochemical staining for HSV-1 and HSV-2 were negative.

\section{Discussion}

The differential diagnosis for multiple ICH includes CAA, hypertensive encephalopathy, CNS vasculitis, arteriovenous malformations and coagulopathy. Hypertension is the most common cause of nontraumatic brain hemorrhage, followed by CAA. The clinical history and laboratory evaluation aid in excluding other diagnostic entities with reasonable certainty. Whereas hypertensive ICH tends to affect deep nuclei of the brain, CAA is a meningocortical angiopathy and therefore shows a predilection for lobar hemorrhage [2]. CAA is most often sporadic, with rare familial forms. Sporadic CAA is associated with Alzheimer's pathology, which this patient showed in the form of beta-amyloid-reactive plaques. Both conditions are 
Shelton et al.: Cerebral Amyloid Angiopathy Presenting with Synchronous Bilateral Intracerebral Macrohemorrhages

characterized by accumulation of $A \beta$, a beta-amyloid precursor protein cleavage product. Accumulation of a shorter variant of the cleavage product is found in CAA (39-40 residues) and a longer variant in Alzheimer's disease (42-43 residues) [5, 6].

The Boston criteria were developed to determine the probability of CAA-related hemorrhage based on imaging findings and clinical data, as tissue is often not available for histologic examination [7]. In patients $\geq 55$ years of age with lobar, cortical or cortico-subcortical hemorrhage and lacking known risk factors or causes for hemorrhage, such as warfarin treatment, head injury or neoplasm, CAA is considered 'probable' in those with multiple hemorrhages and 'possible' in those with a single hemorrhage. Imaging findings in CAArelated hemorrhage may demonstrate asymptomatic microhemorrhages $(\leq 5 \mathrm{~mm})$ or clinically significant macrohemorrhages $(>5 \mathrm{~mm})$ involving the cerebral and/or cerebellar cortices but not the deep gray matter [8]. While our patient did not demonstrate unequivocal microhemorrhages, the presence of bilateral lobar hemorrhages, age $\geq 55$ years and the absence of other identifiable precipitating causes would initially classify this case as 'probable CAA-related hemorrhage', with necropsy serving to confirm the diagnosis.

Lowering of blood pressure likely reduces the risk of CAA-related hemorrhage [9], which corroborates the notion that elevations in blood pressure may act as a precipitant. Furthermore, the current American Heart Association/American Stroke Association guidelines for ICH recommend a target blood pressure of $160 / 90 \mathrm{~mm} \mathrm{Hg}$ and a mean arterial pressure of $110 \mathrm{~mm} \mathrm{Hg} \mathrm{[10]} \mathrm{as} \mathrm{significant} \mathrm{elevations} \mathrm{in} \mathrm{blood} \mathrm{pressure} \mathrm{can} \mathrm{promote} \mathrm{hema-}$ toma expansion. Our patient initially presented with a systolic blood pressure of $178 \mathrm{~mm} \mathrm{Hg}$. It is unclear whether the patient's blood pressure was elevated prior to the hemorrhagic event or whether the hypertension was a component of an evolving Cushing-Kocher response. In either scenario, hypertension seems to have been a plausible initiating and/or exacerbating factor in the development of the patient's large bilateral hemorrhages. It is also known that the patient had been using aspirin $81 \mathrm{mg}$ prior to presentation. The prevalence of cerebral microbleeds and the risk of recurrent ICH are increased in aspirin users with CAA $[11,12]$, suggesting that aspirin use is a risk factor for ICH in CAA and may have also contributed to the severity of the patient's condition.

Multiple ICHs serve as a diagnostic hallmark for the diagnosis of CAA. Interestingly, synchronous bilateral macrohemorrhages are a seemingly rare event and, to our knowledge, have yet to be reported in the literature. We posit that transient elevations in blood pressure in conjunction with aspirin use may be inciting factors for this type of presentation.

\section{Statement of Ethics}

Informed consent for this work was obtained from the decedent's next of kin, and publication of this report was carried out in accordance with Vanderbilt's Human Research Protection Program/IRB committee guidelines.

\section{Disclosure Statement}

The authors declare that no conflict of interest exists in this work. 


\section{Case Reports in Neurology}

Shelton et al:: Cerebral Amyloid Angiopathy Presenting with Synchronous Bilateral Intracerebral Macrohemorrhages

\section{References}

1 Vinters HV: Cerebral amyloid angiopathy: a critical review. Stroke 1987;18:311-324.

-2 Aguilar MI, Freman WD: Spontaneous intracerebral hemorrhage. Semin Neurol 2010;30:555-564.

-3 Yeh SJ, Tang SC, Tsai LK, Jeng JS: Pathogenetical subtypes of recurrent intracerebral hemorrhage: designations by SMASH-U classification system. Stroke 2014;45:2636-2642.

4 Meretoja A, Strbian D, Putaala J, Curtze S, Haapaniemi E, Mustanoja S, Sairanen T, Satopää J, Silvennoinen H, Niemelä M, Kaste M, Tatlisumak T: SMASH-U: a proposal for etiologic classification of intracerebral hemorrhage. Stroke 2012;43:2592-2597.

5 Prelli F, Castano E, Glenner GG, Frangione B: Differences between vascular and plaque core amyloid in Alzheimer's disease. J Neurochem 1988;51:648-651.

6 Suzuki N, Iwatsubo T, Odaka A, Ishibashi Y, Kitada C, Ihara Y: High tissue content of soluble beta 1-40 is linked to cerebral amyloid angiopathy. Am J Pathol 1994;145:452-460.

7 Knudsen K, Rosand J, Karluk D, Greenberg S: Clinical diagnosis of cerebral amyloid angiopathy: validation of the Boston criteria. Neurology 2001;56:537-539.

-8 Chao C, Kotsenas A, Broderick D: Cerebral amyloid angiopathy: CT and MR imaging findings. Radiographics 2006;26:1517-1531.

-9 Arima H, Tzourio C, Anderson C, Woodward M, Bousser MG, MacMahon S, Neal B, Chalmers J; PROGRESS Collaborative Group: Effects of perindopril-based lowering of blood pressure on intracerebral hemorrhage related to amyloid angiopathy: the PROGRESS trial. Stroke 2010;41:394-396.

10 Broderick J, Connolly S, Feldmann E, Hanley D, Kase C, Krieger D, Mayberg M, Morgenstern L, Ogilvy CS, Vespa P, Zuccarello M; American Heart Association; American Stroke Association Stroke Council; High Blood Pressure Research Council; Quality of Care and Outcomes in Research Interdisciplinary Working Group: Guidelines for the management of spontaneous intracerebral hemorrhage in adults: 2007 update: a guideline from the American Heart Association/American Stroke Association Stroke Council, High Blood Pressure Research Council, and the Quality of Care and Outcomes in Research Interdisciplinary Working Group. Stroke 2007;38:2001-2023.

11 Vernooij MW, Haag MD, van der Lugt A, Hofman A, Krestin GP, Stricker BH, Breteler MM: Use of antithrombotic drugs and the presence of cerebral microbleeds: the Rotterdam Scan Study. Arch Neurol 2009;66:714-720.

12 Biffi A, Halpin A, Towfighi A, Gilson A, Busl K, Rost N, Smith EE, Greenberg MS, Rosand J, Viswanathan A: Aspirin and recurrent intracerebral hemorrhage in cerebral amyloid angiopathy. Neurology 2010;75:693698.
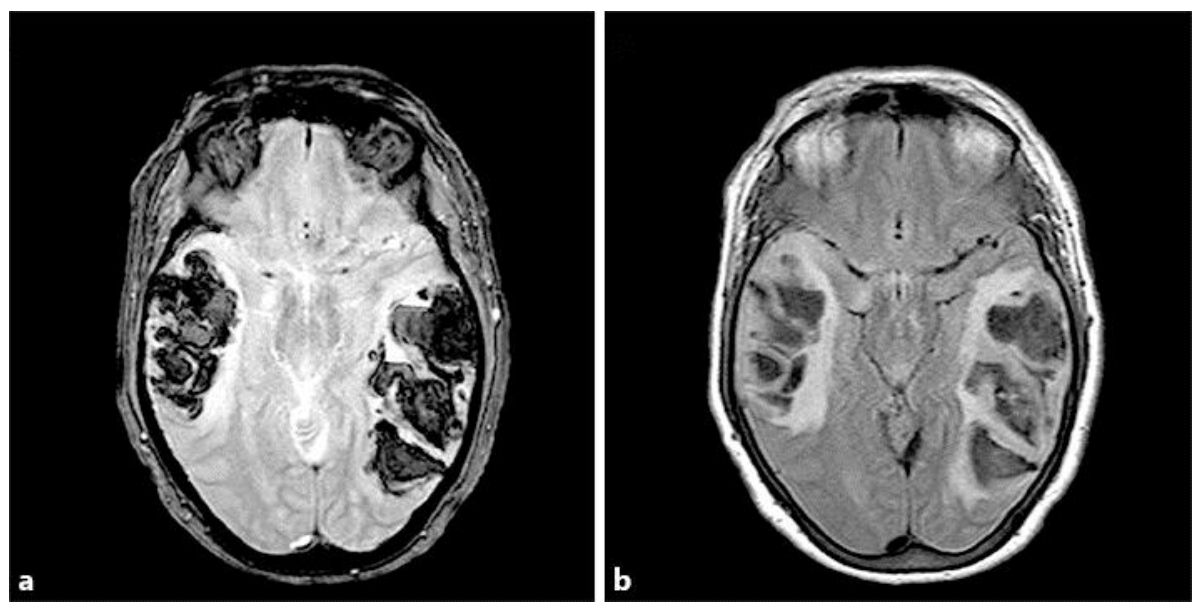

Fig. 1. MRI findings. a Axial T2W FFE image showing large bilateral parenchymal hematomas. b Axial T2W FLAIR image at the same level showing a rim of marked vasogenic edema. 
Shelton et al.: Cerebral Amyloid Angiopathy Presenting with Synchronous Bilateral Intracerebral Macrohemorrhages
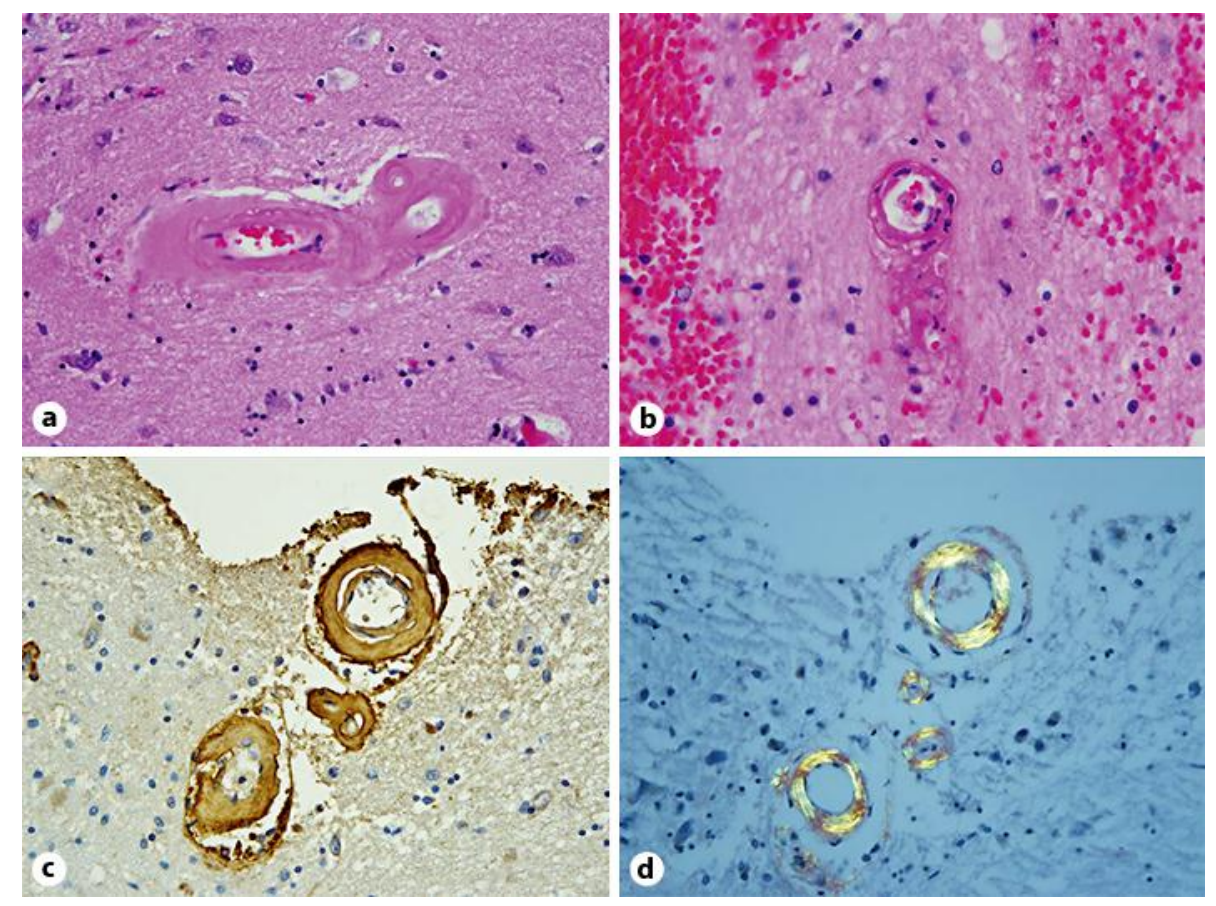

Fig. 2. a Leptomeningeal and cortical arterioles showed thickened, hyalinized walls. b Fibrinoid necrosis indicating CAA-related microangiopathy was seen in blood vessels adjacent to regions of hemorrhage. c Beta-amyloid immunoreactivity was observed in the abnormal arterioles. d Congo red stain viewed under polarized light showed apple-green birefringence. Original magnification: $\times 400$ (a, c, d), ×600 (b). 